\title{
Letramento digital e multimodalidade: uma entrevista com a professora Carla Coscarelli em tempos de pandemia
}

\author{
Carla Viana Coscarelli ${ }^{\mathrm{i}}$ \\ Entrevistadoras: \\ Thayane Verçosa ${ }^{\text {ii }}$ \\ Marcela Santos Brigida ${ }^{\text {iii }}$ \\ Gabriela Ribeiro Nunes ${ }^{\text {iv }}$
}

A impressionante e sólida trajetória da professora Carla Viana Coscarelli, centrada em temas como leitura, produção de textos, ensino e letramento digital, justifica por si só a sua presença como entrevistada neste número da Palimpsesto. Graduada em Letras pela Universidade Federal de Minas Gerais, ela é mestre e doutora em Estudos Linguísticos pela mesma Universidade, tem pós-doutorado em Ciências Cognitivas pela University of California (San Diego) e em Educação pela University of Rhode Island. Sua tese de doutorado, "Leitura em ambiente multimídia e a produção de inferências", defendida em 1999, é um dos exemplos da reflexão da entrevistada sobre a inclusão de múltiplas fontes no processo de leitura e aprendizagem, a qual vem sendo aprimorada ao longo de sua carreira. Atualmente, é Professora Titular da Universidade Federal de Minas Gerais, onde participa do Núcleo de Pesquisa Lingtec (Língua e Tecnologia), do Ceale (Centro de alfabetização, leitura e escrita) e coordena o Projeto de Extensão Redigir, um belíssimo

\footnotetext{
i Doutorado em Estudos Linguísticos pela Universidade Federal de Minas Gerais (1999), pós-doutorado em Ciências Cognitivas pela University of California San Diego (2005) e pós-doutorado em Educação pela University of Rhode Island. Professora Titular da Universidade Federal de Minas Gerais.

ii Doutoranda em Literatura Brasileira pela Universidade do Estado do Rio de Janeiro (UERJ). Bolsista CAPES. thayanevercosa@ hotmail.com

iii Doutoranda em Literaturas de Língua Inglesa pela Universidade do Estado do Rio de Janeiro (UERJ). Bolsista CNPq. marcela.brigida@uerj.br

iv Doutoranda em Teoria da Literatura/Literatura Comparada pela Universidade do Estado do Rio de Janeiro (UERJ).j15c17g11 @ hotmail.com
} 
exemplo de como ensino, pesquisa e extensão, quando bem articulados, geram resultados muito frutíferos.

É com muita satisfação que publicamos esta entrevista com a professora Carla Coscarelli, que prontamente aceitou o nosso convite, sendo muito receptiva às reflexões que lhe propusemos. Gostaríamos de destacar que foi um prazer conhecer a inspiradora trajetória da professora e aprender com a sua produção tópicos tão relevantes e atuais, ainda mais no presente contexto de difusão global do ensino remoto em caráter emergencial.

\section{PALIMPSESTO}

1) Há 11 anos, na sua entrevista concedida ao periódico Educação \& Tecnologia, a pergunta "Como ficam a alfabetização e o letramento em tempos de web e de redes sociais digitais?" foi respondida da seguinte forma: "Acho que tanto a alfabetização quanto o letramento podem se dar bem em tempos digitais. A web hoje é muito dependente da escrita e da leitura. Isso pode mudar, mas, atualmente, precisamos ser alfabetizados e letrados para lidar com desenvoltura com o computador e a web. Isso cria uma demanda e as pessoas tendem a reagir a essas demandas sociais, aprendendo o que precisam saber, seja na escola, seja com os amigos e parentes. A necessidade de ler e escrever pode criar o desejo de aprender, e ter situações reais que demandam a leitura e a produção de textos vai ajudar no desenvolvimento das habilidades necessárias aos dois processos. Acredito que a escola esteja se preparando para ajudar as pessoas a lidarem bem com os ambientes e espero que isso, em breve, não seja um privilégio das classes mais altas, mas seja também uma realidade das camadas populares" (COSCARELLI, 2009, p. 7). Tendo em vista o que mudou ao longo de mais de uma década e considerando que de lá para cá a presença da web e das redes sociais no cotidiano se intensificou, como você responderia hoje aquela mesma questão?

\section{CARLA COSCARELLI}

Por um lado, é bom dizer que eu responderia praticamente da mesma forma. $\mathrm{O}$ letramento e a alfabetização, que é uma parte desse processo interminável de conquistar o estado ou a condição adquirida por quem se apropria da escrita (Madga Soares, 1998), são muito importantes em nossa sociedade. Os computadores, incluindo nessa palavra os celulares, que, na verdade, são computadores de bolso, fazem parte da nossa vida e a escrita é requerida na maioria das ações que realizamos nesses equipamentos digitais. 
Acrescentaria aí o trabalho com a multimodalidade, ou seja, com as várias linguagens, além da necessidade cada vez maior de termos um olhar crítico e atento a todas as informações que encontramos, para que possamos detectar e lidar com fake news, para evitar cair em golpes, que estão cada vez mais sofisticados.

A alfabetização deve ser vista de uma forma ampla, porque precisa incorporar a escrita e a leitura nesses equipamentos, como, por exemplo, saber usar o teclado, se familiarizar com os programas de produção de textos, como os editores de texto (como o Word), programas de apresentação (como o PowerPoint), programas para fazer infográficos, banners, postagens nas redes sociais, planilhas.

A produção desses textos demanda saber usar equipamentos (computador, celular, teclado, mouse, programas, aplicativos) que precisam ser conhecidos e, na medida do possível, dominados pelos usuários. O computador é nosso caderno e precisamos aprender a escrever nele, explorando os recursos que ele nos oferece.

As tecnologias digitais de informação e comunicação trouxeram novos espaços de compartilhamento de textos, novos espaços de leitura e de escrita, formas de comunicação em que se compartilham áudios, vídeos, animações, imagens, fotografias, entre outros, então, saber receber (ler, ver, assistir, apreciar, criticar, etc.), saber produzir e saber compartilhar esses textos são competências que precisamos ter como cidadãos de nossa sociedade contemporânea. Negar isso às pessoas é negar acesso à informação e, de alguma forma, é também negar o direito de expressão, o que é contra a lei.

Por outro lado, é triste dizer que a minha resposta para esta pergunta continua praticamente a mesma. Depois de mais de uma década, eu gostaria de poder dizer que as escolas se prepararam e hoje as pessoas sabem usar bem os ambientes digitais, são boas leitoras e produtoras dos gêneros textuais que circulam neles e sabem lidar bem com as diversas linguagens que eles demandam ou propiciam. Eu gostaria de dizer também que o acesso aos equipamentos digitais e à internet é igual para todos e que a escola é um lugar onde se aprende a lidar com eles de forma respeitosa, ética, criativa e emancipadora. Infelizmente, vou ter de continuar desejando que a escola prepare as pessoas para lidar bem com esses ambientes e defender que precisamos lutar muito para que o acesso aos equipamentos e à informação deixe de ser um privilégio das camadas mais privilegiadas da nossa sociedade e seja um direito (respeitado e cumprido) de todos. 


\section{PALIMPSESTO}

2) Em tempos de pandemia e de isolamento social, no qual as nossas formas de interação social, de trabalho e de ensino foram direta e, talvez, irreversivelmente impactadas, qual é a sua opinião sobre a atual implementação maciça do ensino a distância, considerando que este, na maioria dos casos, tem consistido em uma tentativa de transferir a estrutura da sala de aula para as diversas plataformas digitais? É possível pensar em vantagens e desvantagens dessa transferência?

\section{CARLA COSCARELLI}

Primeiramente, precisamos fazer uma distinção entre ensino a distância $(\mathrm{EaD})$ e ensino remoto emergencial (ERE). EaD tem uma regulamentação, são (ou deveriam ser) cursos bem planejados, em que um ambiente virtual de aprendizagem (AVA) é escolhido para que as aulas aconteçam nele, os professores são preparados para esse ensino, sabem lidar com os AVA, planejam estratégias didáticas para abordar os assuntos nessa modalidade de ensino, planejam as formas de interação com os alunos em cada atividade, equilibram atividades síncronas e assíncronas dependendo das demandas do curso e das turmas. Esses professores sabem de antemão que precisam contar com determinados equipamentos como um bom computador, microfones, câmeras, precisam contar com uma conexão de internet boa e estável. Além disso, sabem lidar com programas para gravar e editar aulas ou contam com uma estrutura para auxiliar essas gravações e edições de áudios e/ou vídeos, conhecem e sabem criar atividades, avaliações, formas de interação entre os participantes, ou seja, sabem planejar e gerenciar espaços virtuais de aprendizagem. Os alunos, por sua vez, sabem que as aulas serão a distância e por isso precisam ter os equipamentos para acessar as atividades, para realizar as tarefas e para participar das interações. Os alunos também precisam ser aprendizes organizados e disciplinados e, ao se matricular, sabem e aceitam que a disciplina será na modalidade a distância. É uma escolha. Existe uma preparação e um acordo pré-estabelecido entre os que participam da $\mathrm{EaD}$.

O ensino remoto emergencial, por sua vez, foi fruto de uma situação de impedimento dos encontros presenciais provocado pela pandemia. De uma hora para a outra, os cursos presenciais não puderam ser ministrados nesse formato e outras formas de contato com os alunos tiveram de ser criadas. Algumas escolas e algumas comunidades puderam usar quase que prontamente os computadores para continuar o trabalho. Outras 
não puderam fazer isso porque alunos e professores não tinham equipamento e recursos para que essa transferência de uma modalidade para a outra acontecesse. É uma questão delicada, reflexo de uma sociedade de diferenças socioeconômicas tão grandes entre as pessoas e que demanda muita discussão sobre questões estruturais de nossa sociedade e de políticas públicas. Então, vou me ater à sua pergunta: tentar transferir exatamente o que acontece na aula presencial para as plataformas digitais é um grande equívoco, porque as dinâmicas precisam ser diferentes. Na sala de aula presencial o professor, mal ou bem, consegue dar uma aula expositiva de 40 minutos. No online, os alunos vão desligar as câmeras e vão fazer outras coisas. Na aula presencial os alunos trocam ideias com o colega do lado, conversam entre uma aula e a outra, tem o relaxamento e as interações com os colegas na hora do intervalo. Então, precisamos pensar em formas de fazer com que as interações entre os alunos continuem acontecendo.

Aulas em ambientes digitais precisam ser pensadas para esse formato que possibilita, por exemplo, que as atividades sejam assíncronas (Síncrono - todo mundo no mesmo "lugar" ao mesmo tempo / Assíncrono - cada um trabalha no horário que melhor lhe convier). No presencial, o que aconteceu não volta mais. No online, posso assistir a um vídeo várias vezes, posso realizar a atividade em outros horários que não seja o estipulado pela escola. Essa possibilidade precisa ser considerada, porque não sabemos se na casa tem dois alunos com aulas pela manhã e só tem um computador disponível, por exemplo. Nesse caso, alguém sairia prejudicado. Não precisamos fazer chamada porque o AVA me diz quem entrou no ambiente e que atividades esse aluno realizou nele. Lá mesmo o professor pode ler os trabalhos feitos pelos alunos, enviar feedback, dar nota, outros alunos podem ler e comentar os trabalhos dos colegas e, se for o caso, o aluno pode modificar o que produziu incorporando os comentários recebidos e repostar sua produção. São dinâmicas que podem ser exploradas no ensino assíncrono e que não dependem de estar todo mundo junto em uma sala de aula virtual na mesma hora Encontros síncronos também podem ser interessantes, produtivos e enriquecedores, mas isso depende do que será feito nele. Longas aulas expositivas e uma dinâmica centrada no professor talvez não seja a forma mais produtiva de usar o tempo das atividades síncronas. O tempo com os alunos (que não precisa todo o tempo computado na carga horária do curso) deveria ser usado para conversar sobre as tarefas, para os alunos trazerem e discutirem dúvidas, para compartilharem resultados. A partir de uma primeira conversa e de uma proposta feita e 
negociada com a turma em um encontro síncrono, os grupos de alunos podem, no tempo das atividades assíncronas, realizar a tarefa conversando, escrevendo e produzindo colaborativamente o trabalho usando para isso ambientes digitais (ex. Whatsapp, Google docs, ferramentas disponíveis no AVA adotado).

Tenho na minha cabeça e nas minhas práticas uma concepção de aprendizagem em que os alunos realizam atividades para aprender sob a orientação do professor. Gosto e sei que é muito produtiva uma educação centrada no aluno.

\section{PALIMPSESTO}

3) No artigo publicado com a professora Ana Elisa Ribeiro, "Leitura e ensino: por avaliações que levem (mesmo) os ambientes digitais em consideração" (2019), ao refletir sobre o crescimento e a relevância da leitura em ambientes digitais, bem como justificar a necessidade de que as práticas de leitura em tais ambientes sejam incluídas nos currículos escolares, vocês refletem: "A maioria dos adolescentes parece nunca ter aprendido o que fazer para julgar a confiabilidade das informações que encontra na internet [...], uma vez que não usam estratégias eficientes para avaliar esse quesito" (COSCARELLI; RIBEIRO, 2019, p. 103). Em que medida esta lacuna educacional se relaciona com a difusão das fake news e do seu impacto negativo no cotidiano das pessoas? Além da inclusão dessas práticas nos currículos escolares, podemos pensar em outras medidas que auxiliem na construção da capacidade de "julgar a confiabilidade das informações"?

\section{CARLA COSCARELLI}

A questão das fake news é complexa, porque há a disseminação de notícias falsas, mas há quem se beneficie e quem apoie a divulgação dessas notícias porque elas refletem e reforçam uma ideologia, as ideias de um determinado grupo, um conjunto de comportamentos que algumas pessoas querem reforçar, etc. Mas, de qualquer forma, ser um leitor crítico e atento tem relação direta com a disseminação de fake news, uma vez que esse tipo de leitor pode ajudar a diminuir a disseminação dessas notícias enganosas e pode levar outras pessoas a examinar com mais cuidado as informações que recebem.

As pessoas precisam analisar as informações considerando quem disse aquilo, em que situação, se aquela pessoa tem competência e expertise para tratar daquele tema, a quem interessa aquela informação, que vozes ou aspectos são silenciados nele, ou seja, têm de pensar sobre aquele material com cuidado e um certo grau de exigência. Tem de 
ser desconfiado e buscar o que há por trás daquela fala, o que há nas entrelinhas, por que aquilo foi dito daquela forma, se aqueles argumentos são robustos, se são apresentadas evidências, se aquelas informações são verdadeiras, quais seriam as fontes daquelas informações, entre muitas outras perguntas que podem ser feitas.

As pessoas precisam aprender a ler criticamente, a refletir sobre as informações que encontram ou recebem e avaliar a pertinência daquela informação antes de repassálas para outras pessoas. Isso envolve uma leitura que podemos chamar de mais profunda em contraposição àquela leitura superficial e sem reflexão. Nenhuma leitura deveria ser superficial, não é mesmo? Não há texto despretensioso nem neutro, não há discurso que não seja retórico e argumentativo. Então, o leitor tem de se acostumar a fazer esse exercício de leitura desconfiada.

A capacidade de avaliar a confiabilidade das informações deve ser sempre estimulada pelas escolas, pelos pais, e também em campanhas pelos mais diversos meios de comunicação. É uma questão de cidadania e de segurança, pois essa leitura desconfiada também pode servir como prevenção contra golpes e crimes que são cada vez mais numerosos nos ambientes digitais.

\section{PALIMPSESTO}

4) No texto publicado com Luana Lopes Amaral, "O ensino de escrita acadêmica a distância na UFMG: um relato de experiências" (2017), ficam evidentes os resultados positivos da disciplina "Oficina de Língua Portuguesa: Leitura e Produção de Textos", ministrada na modalidade a distância (através do Moodle) e ofertada a todos os cursos de graduação da UFMG há mais de uma década. Ao agradecer as parcerias firmadas com diferentes órgãos da Universidade, vocês dizem: “Ainda há muito preconceito e ideias equivocadas com relação ao ensino a distância, e esse apoio de instâncias superiores da universidade tem nos ajudado a mostrar que essa modalidade de ensino é tão eficiente e idônea quanto o ensino presencial - vários dados de pesquisas apontam para esse fato [...]. Além disso, tem nos possibilitado explorar as diversas potencialidades desse ensino, desenvolvendo um trabalho de ponta na universidade e que poderá servir de modelo e de experiência para outros professores e cursos" (COSCARELLI; AMARAL, 2017, p. 194). $\mathrm{Na}$ sua opinião, a que se deve o referido preconceito com relação ao ensino a distância? Em que medida a "Oficina" contribuiu para superá-lo na universidade em que você trabalha? Você tem conhecimento de iniciativas análogas influenciadas ou não pela "Oficina"? 


\section{CARLA COSCARELLI}

Não posso afirmar que todo curso a distância seja ótimo, assim como não posso defender que todo curso presencial seja de boa qualidade. Sabemos que há cursos bons e ruins nas duas modalidades.

O preconceito em relação à $\mathrm{EaD}$ atualmente vem de má informação sobre como esses cursos acontecem. No entanto, se pensarmos no histórico do ensino a distância esse preconceito talvez tenha vindo da lentidão das trocas de informação pelo correio de antigamente nos cursos que eram feitos por correspondência. As aulas custavam a chegar ao aluno, a resposta dele demorava a chegar ao professor, o feedback e as avaliações dos trabalhos chegavam quando o aluno provavelmente nem estivesse se lembrando mais do que enviou por causa lentidão das formas de comunicação. Além disso, era uma aprendizagem solitária, sem uma turma ou um grupo de pessoas que trocassem ideia rápida e facilmente. $\mathrm{O}$ aluno precisava ter muita motivação e paciência para aprender, então, muita gente desistia, o que acabava sendo visto como um problema do curso. Podemos considerar também fatores econômicos, ou seja, quem tinha dinheiro ia para a universidade, quem tinha poucos recursos se contentava com cursos a distância por correspondência ou por rádio para fazer um curso profissionalizante. Então, por uma série de fatores, a EaD ficou estigmatizada como uma modalidade de ensino menor, pior, pouco eficaz, mas, na verdade, sabemos que ela atendeu milhões de alunos que aprenderam suas profissões ou algum ofício (Instituto Universal Brasileiro, Rádio-Escola Municipal no Rio, entre outros).

Com a internet, o cenário para a $\mathrm{EaD}$ muda. A comunicação se torna rápida e é cada vez mais acessível para um número maior de pessoas, o que faz com que a formação de grupos de interesses seja fácil, assim como os encontros síncronos ou a troca de mensagens e materiais de forma assíncrona. Isso faz com que os cursos possam ser mais dinâmicos, possam explorar diversos tipos de materiais como textos, áudios, vídeos, animações, fóruns de discussões, entre tantos outros recursos que podem ser usados com facilidade.

A Oficina de Leitura e Produção de Textos trabalha com a inserção dos alunos no universo acadêmico e, para isso, apresenta o Currículo Lattes e o Portal de Periódicos da Capes aos alunos, discute as diferentes formas de citar trabalhos e a gravidade do plágio na academia. Passa pela produção de resumos, de resenhas, a escrita de um projeto, a 
coleta de dados, a análise desses dados e a elaboração de um pequeno relatório de pesquisa. Essa é uma formação inicial importante para os alunos e acaba tendo impacto positivo em toda a formação deles na universidade, como os próprios alunos relatam. $\mathrm{O}$ material e a dinâmica da Oficina têm sido construídos e melhorados a cada semestre durante mais de 10 anos. Sendo assim, acabamos chegando a uma forma de trabalho bem robusta (e nem por isso engessada) e que tem avaliação muito positiva dos alunos. Atendemos 500 alunos de diversos cursos da UFMG por semestre e temos uma equipe com professores e tutores muito dinâmica e bem entrosada.

$\mathrm{Na}$ Faculdade de Letras da UFMG temos outros projetos muito bem-sucedidos como o ensino de Inglês (Ingrede), que começou na mesma época da nossa Oficina, e o ensino de Libras, que é um pouco mais recente. Há muitos outros projetos bonitos de EaD espalhados pelo Brasil. A Universidade Aberta do Brasil, por exemplo, é um programa que ampliou muito a oferta do ensino a distância para o ensino superior. Precisamos torcer para que não acabem com ela.

\section{PALIMPSESTO}

5) No texto "O que dizem as matrizes de habilidades sobre a leitura em ambientes digitais" (2010), você e a professora Ana Elisa Ribeiro discutiram "aspectos da matriz de Língua Portuguesa utilizada como base para a composição das provas do ensino básico", refletindo, em larga medida, sobre "como essa matriz é [i] impregnada de valores ligados à cultura escrita impressa; [ii] forjada com base em pressupostos que não levam em consideração a multimodalidade dos textos que circulam na sociedade; [iii] redigida de maneira imprecisa em grande parte dos descritores; e [iv] carente de uma ampliação para que agregue elementos importantes para uma avaliação menos vaga de habilidades sustentadas por experiências de leitura em ambiente digital" (COSCARELLI; RIBEIRO, 2010, p. 318). Uma década depois, as matrizes de habilidade sobre a leitura passaram a incluir elementos de avaliação que efetivamente contemplem a leitura em ambiente digital? Quais foram os avanços? E os desafios ainda a serem enfrentados?

\section{CARLA COSCARELLI}

Podemos dizer que a Base Nacional Curricular (BNCC) foi um avanço, no que diz respeito à inclusão do digital nas propostas pedagógicas. A Base expressa uma preocupação com a diversidade e com uma formação dos sujeitos para exercer a cidadania. A Base fala explicitamente nas tecnologias digitais de informação e 
comunicação (TDIC) e menciona muitos gêneros digitais como meme, vlog, podcast, charge digital, entre outros.

Além disso, a BNCC fala em navegação, embora sem mencionar essa palavra. Fala em busca e seleção de informações e menciona inclusive a curadoria, que podemos entender como a construção e organização de um acervo ou de um repertório com informações que vão ser úteis ou necessárias para um determinado propósito. Fala em selecionar informações em fontes confiáveis tanto impressas quanto digitais. Esse documento apresenta as habilidades importantes para a lida com as tecnologias digitais como um aspecto transversal, que atravessa diversas áreas e temas. Isso é muito bom.

A Base menciona muitos gêneros textuais que vão demandar o uso de equipamentos como gravadores, filmadoras, câmeras, microfones, editores de áudio, vídeo e textos escritos, e isso tudo está no celular, que é proibido em muitas escolas. Então, acho que a BNCC nos obriga a repensar a escola e os seus modos de fazer a educação, puxando para uma educação mais contemporânea, que vai ensinar os alunos a usar os equipamentos que trazem no bolso para aprender, para produzir conteúdo e compartilhar suas criações.

A BNCC também apresenta uma preocupação com a multimodalidade, com a exploração de outras linguagens além da verbal. O documento menciona explicitamente práticas que envolvem a recepção, a produção e a interação feita com gêneros textuais multissemióticos e multimidiáticos. Isso é um avanço em relação a outras matrizes de avaliação como, por exemplo, a do Sistema de Avaliação do Ensino Básico (SAEB).

Sabemos, no entanto, que a teoria nem sempre se realiza na prática. Para que as ideias apresentadas na Base se concretizem, é preciso que os profissionais da educação recebam formação para isso e que tenham condições de colocar essas ideias em prática. É fundamental que a comunidade escolar possa contar com bons equipamentos, bom acesso à internet (inclusive em casa, onde parte do trabalho é realizada tanto por professores quanto por alunos). Essa pandemia nos mostrou que estamos muito longe de ter o básico para pensar uma educação para tempos digitais, sobretudo no ensino público, que é mais de $70 \%$ da população escolar no Brasil.

\section{PALIMPSESTO}

6) O Projeto Redigir, coordenado por você, desde 2009 disponibiliza, para serem usadas nas aulas de Língua Portuguesa da educação básica, atividades alinhadas com as teorias 
contemporâneas dos estudos linguísticos, literários, discursivos e da educação. Como se dá, efetivamente, a articulação entre teoria e prática na elaboração dessas atividades? $\mathrm{O}$ projeto leva em consideração as mudanças nos documentos oficiais do Ministério da Educação voltados para a definição de parâmetros para o ensino da Língua Portuguesa no Brasil? Nesses mais de dez anos de atividade, como têm se dado o contato e a interlocução entre a Universidade e a comunidade escolar que se serve dos materiais produzidos pelo projeto?

\section{CARLA COSCARELLI}

O Redigir completou em 2020, 21 anos de atividades. Nesse projeto, nós procuramos colocar em prática uma concepção de ensino de Língua Portuguesa mais voltado para o letramento, ou seja, para o uso das linguagens e não para a metalinguagem pura e seca como acontece com o ensino normativo da gramática tradicional, que ainda é muito comum em nossas escolas.

Criamos atividades para serem aplicadas pelos professores, pensando em temas, gêneros textuais e discursivos que são importantes para a formação de um cidadão contemporâneo. Sendo assim, trabalhamos com temas como a diáspora, as cidades, as etnias, o racismo, a violência contra a mulher, o consumo, os relacionamentos, a arte, pichação, grafite. São muitos assuntos. Trabalhamos com diversos gêneros textuais como canções, curtas-metragens, poemas, contos, crônicas, embalagens de produtos, pontos de venda, anúncios publicitários, notícias, entre muitos outros. É um projeto que procura tratar os temas de forma leve e interessante para os alunos e para os professores, sem deixa de discutir os temas com seriedade e profundidade.

Buscamos desenvolver habilidades relacionadas ao letramento digital e, para isso, trazemos atividades que lidam com a busca, seleção, análise, síntese integração e uso de informação provenientes de múltiplas fontes, com o uso de tecnologias digitais disponíveis gratuitamente na internet.

Estudamos teorias de várias áreas e que são importantes para pensar sobre a vida, são teorias que nos ajudam a entender a educação, as linguagens, as pessoas, as sociedades, a mídia, o universo digital e a influência dele nas nossas vidas. Estando atentos a essas teorias, podemos pensar em formas de trabalhar os textos com os alunos, trazendo a exploração interdisciplinar dos temas e apontando aspectos que precisam ser percebidos, analisados e avaliados pelos alunos. As teorias ajudam a orientar nosso olhar. 
Por exemplo, temos conosco sempre teóricos inspiradores de diversas áreas, como Magda Soares, Paulo Freire, Celestin Freinet, Mimi Ito, Sugata Mitra, Jean François Rouet, Jacques Ranciere, Ailton Krenak, Djamila Ribeiro, Gunther Kress, Edgar Morin, Roxane Rojo, Ana Elisa Ribeiro, Mário Perini, John Dewey, Vygotsky, Wlakiria Monte Mór, Julie Coiro, Renée Hobbs, Roxane Rojo, entre outros. É uma turma enorme de pesquisadores e pensadores que nos inspiram e é uma lista que cresce a cada dia porque estamos sempre lendo, pesquisando e buscando contribuições dos estudos de diversas áreas.

A teoria dos multiletramentos, que foi proposta pelo Grupo de Nova Londres (Cazden et al., 1996), nos lembra que precisamos pensar nas múltiplas linguagens orquestradas em um texto e que os textos são sempre multimodais (Kress, van Leeuwen, 1996, 2002; Ribeiro, 2016). Mesmo quando parecem ser feitos apenas de palavras, existe ali uma escolha da fonte, das cores, da diagramação, do espaçamento, da formatação de modo geral, que, em si, já trazem sentidos a serem construídos pelos leitores. Os programas e aplicativos disponíveis em ambientes digitais trazem muitas possibilidades para o trabalho com várias linguagens, fazendo com que seja cada vez mais simples explorar recursos visuais nos textos, produzir e combinar áudios, vídeos, animações.

Os multiletramentos também chamam nossa atenção para a diversidade cultural das nossas sociedades, que precisa ser compreendida e respeitada. Vou dar o exemplo da variedade linguística. Estamos acostumados a pensar que no Brasil falamos todos a mesma língua. Isso não é uma verdade, temos diversas línguas indígenas, quilombolas, em algumas comunidades se falam línguas orientais, em outras se falam línguas europeias ou dialetos delas. E mesmo dentro do português, temos uma gama enorme de registros e de variantes que precisam ser conhecidos, respeitados e desestigmatizados. O preconceito linguístico existe, gera discriminação, e, como todos os tipos de preconceito (religioso, racial, social, entre outros), precisa ser combatido, por que é uma forma de colocar algumas pessoas em posição de inferioridade para que outras assumam o lugar de superiores, desencadeando um processo de poder muito cruel.

Em muitas situações, existe uma tentativa de apagar o sotaque das pessoas (veja por exemplo a mídia televisiva / as gravadoras). Isso é de uma violência silenciosa e simbólica muito grande. É o silenciamento de vozes, de culturas, das diferenças para a instalação de um "padrão superior" que faz com que tudo o que seja diferente daquilo 
seja percebido como inferior, ruim, digno de ser ignorado e exterminado. É medonho, desumano e muito perigoso.

A noção de multiletramentos nos é muito cara porque nos dá uma visão de que somos diversos, múltiplos, que diferentes culturas se manifestam em diferentes combinações de linguagens e é muito empobrecedor eleger apenas uma forma ou uma cultura como sendo a que precisa prevalecer sobre todas as outras.

Preferimos a emancipação ao embrutecimento (Ranciere, 2002, Krenak, 2019). Por isso, em nossas atividades buscamos formas de dar autonomia para os alunos, trabalhando o questionamento, o olhar crítico, procuramos dar voz a eles e desenvolver formas de empoderamento, de compreensão dos fenômenos sociais, de combate aos preconceitos, etc.

São muitas teorias em jogo, não tenho espaço aqui para falar de todas elas, mas espero ter mostrado o espírito das teorias que orientam nosso trabalho.

Pensamos muito nos professores também, as atividades são uma forma de desenvolver a formação continuada deles. Todas as nossas atividades têm uma parte para o professor bastante detalhada, trazendo um diálogo da nossa equipe com eles, mostrando possibilidades de respostas, orientações, apresentando possibilidades e explicitando o que pretendemos com aquelas questões. Nos esforçamos para que as atividades ajudem os professores a sair do tradicional e a pensar que o ensino de língua materna pode ser feito de outras formas, que a reflexão linguística pode (e deve) ser feita fora das amarras e das inconsistências da gramática tradicional, pode ser feita de forma contextualizada e significativa para os alunos.

Recebemos cada vez mais respostas e depoimentos de professores que usam nossas atividades. Recebemos também sugestões de temas para as atividades. Procuramos perceber e atender as demandas deles. No início da pandemia, por exemplo, percebendo que muitos professores estavam tensos, cheios de dúvidas e questionamentos, fizemos uma série de dicas que mostravam aplicativos e programas que eles poderiam usar com os alunos e dávamos sugestões de como fazer isso. Essas dicas foram reunidas em um ebook que está disponível para os professores gratuitamente (como tudo que disponibilizamos para eles) no nosso site (www.redigirufmg.org).

Temos um diálogo intenso com os professores pelas redes sociais. Com a pandemia e o isolamento físico, recebemos muitos convites para dialogar com professores 
de escolas de diversas regiões do país, fizemos muitas "lives" que foram conversas muito ricas com os professores. A educação enfrenta uma situação ainda mais desafiadora nessa pandemia. Isso daria mais muitas páginas de conversa, que vou resumir dizendo que a universidade e a comunidade são muito mais parceiras do que as pessoas imaginam. A universidade pública tem inúmeras parcerias com a comunidade tanto diretamente pelos seus projetos e programas de extensão, quanto pelas pesquisas que geram impacto positivo na vida das pessoas. Isso, sem falar do ensino, que completa o nosso tripé (ensino, pesquisa e extensão), e que devolve para a sociedade profissionais capacitados para trabalhar nas mais diversas áreas. Sim, sou uma apaixonada e entusiasta da universidade pública. Sou fruto do ensino público, acredito e defendo o ensino público e de alta qualidade para todos.

\section{PALIMPSESTO}

7) O seu projeto de pesquisa mais recente "Realidade aumentada: buscando formas de promover a leitura e a aprendizagem”, iniciado em 2019, é descrito por você da seguinte maneira: "O principal objetivo desse projeto é desenvolver formas de usar a realidade aumentada para promover a aprendizagem autônoma e a leitura crítica. Para isso, vamos produzir materiais de compreensão de textos que servirão de guia para os leitores. Esses materiais buscam trabalhar habilidades de leitura que são essenciais para a compreensão de textos multimodais de diversos gêneros e domínios discursivos" (Fonte: Currículo Lattes). O que é a realidade aumentada e como ela pode auxiliar na promoção da aprendizagem autônoma e da leitura crítica? Como serão os materiais que servirão de guia para os leitores? Como funciona, na prática, a leitura aumentada?

\section{CARLA COSCARELLI}

Temos muita tecnologia digital disponível e precisamos pensar nas potencialidades delas. No meu caso, procuro formas de melhorar as habilidades de leitura das pessoas, de ajudar os alunos a serem leitores fluentes, críticos e competentes. A realidade aumentada é, a grosso modo, uma forma de integrar o virtual e o real. É uma forma de enriquecer ou complementar "o ambiente real com objetos virtuais, usando algum dispositivo tecnológico, funcionando em tempo real”. Essa é uma definição de Kirner e Siscoutto (2007, p. 10).

É uma tecnologia que ativa 'links' a partir de imagens, é uma ampliação do hipertexto. O QR code, por exemplo, é uma forma simples de realidade aumentada. 
Aquela imagem te leva a outros lugares. O Pokemon Go, que fez um sucesso enorme, usa realidade aumentada. A partir de um gatilho - uma imagem reconhecida por uma câmera ou sensor, que, por sua vez pode estar no celular, num tablet, em uns óculos - você pode ativar um áudio, uma animação, um filme, e outras fontes de informação. Isso gera possibilidades enormes. Você pode, a partir do reconhecimento da fachada de uma casa, ver uma personagem contando algum fato histórico que tenha acontecido ali. A partir de um cartaz, você pode ver o artista falando sobre o espetáculo e te convidando para assistir. Tendo a imagem de uma célula como gatilho, você pode apreciar as formas de reprodução dela. Ao identificar uma obra de arte com seu celular, você pode ver o artista produzindo aquela obra, falando dela, ou obter alguma informação sobre o artista, a época e as circunstâncias em que ela foi produzida, assim como algumas dicas de como interpretar ou compreender melhor aquela arte. A partir de um grafite, você pode ampliar e animar aquela imagem e "entrar" nela, experienciando, de forma muito real e corpórea, aquela nova realidade, como é o projeto artístico Aquário Urbano feito por Felipe Yung Maciel (Flip) e pelo Kléber Pagu, que desenvolveram um aquário virtual a partir de grafites feitos em prédios de São Paulo. Um projeto maravilhoso.

O potencial dessa tecnologia é enorme nas mais diversas áreas do conhecimento e pode ser um forte aliado na educação. Os alunos podem aprender muito com realidade aumentada e podem produzir materiais usando esse recurso, o que vai gerar um conhecimento interdisciplinar muito enriquecedor.

A Tamara Milhomem desenvolveu um projeto muito interessante na biblioteca da UFPI, com seus alunos do Instituto Federal do Piauí - Campus Picos, unindo a vontade de incentivar a leitura, de desenvolver habilidades de interpretação de textos e a realidade aumentada. Ela incentivou os alunos a criarem realidade aumentada a partir de capas de livros que eles leram. Os alunos selecionaram as obras, leram, discutiram e criaram a realidade aumentada - a partir da capa, da contracapa ou da lombada do livro - que acionava uma produção desses alunos criada a partir do livro, como forma de motivar outros alunos a ler aquela obra. Foi um sucesso.

Estou desenvolvendo junto com a equipe do Interaction da USACH, sob a coordenação do professor Roberto Gonzáles Ibañez, um projeto que usa a realidade aumentada para desenvolver habilidades de leitura, que consiste em integrar o impresso e o digital. A partir do reconhecimento do texto e de movimentos feitos com mãos, o 
leitor pode complementar o texto com informações extras, como o significado de palavras, verbetes de enciclopédia, imagens, animações, filmes ou áudios explicativos, pode fazer anotações que serão armazenadas no computador e/ou ver as anotações feitas por outras pessoas, pode selecionar e guardar partes do texto, entre outras possibilidades.

Estamos desenvolvendo também outro projeto em que o leitor usa o celular para, a partir do texto impresso, ativar imagens, animações e áudios que trazem perguntas ou informações sobre elementos encontrados naquela página, explorando as imagens do texto assim como a parte verbal dele. Assim, o aplicativo de realidade aumentada vai mostrando para o aluno as perguntas que ele pode fazer aos textos, as informações que ele pode querer procurar fora dele, pode perceber a importância de articular os recursos verbais e visuais, as inferências que ele pode ou precisa fazer para enriquecer a leitura e os questionamentos que vão levar a uma leitura crítica dos materiais que ele encontra nos mais diversos ambientes e situações comunicativas. Assim, de forma leve, procuramos desenvolver a competência de leitura dos sujeitos. A leitura aumentada, como gostamos de chamar, pode ser usada por pessoas de todas as idades, desde que se façam materiais adequados para aquela faixa etária e para aqueles determinados propósitos e temas.

Isso tudo pode ser usado para fins educacionais e para promover a aprendizagem. O usuário, de posse de um material desses, pode aprender a aprender. Gostaria de lembrar que a figura do professor é sempre importante, pois ele vai promover as discussões, vai orientar os alunos em suas aprendizagens, vai organizar o trabalho, vai selecionar os temas e pensar em uma gradação e dificuldades, vai lançar desafios. Um programa desses não dispensa o professor, muito pelo contrário, o professor é uma peça importante para orientar os alunos no uso e na criação desses materiais.

Acho muito importante que os alunos façam coisas, produzam materiais, sejam ativos na aprendizagem, por isso, o professor deve jogar na mão dos alunos a possibilidade de eles mesmos construírem suas formas de aprendizagem e uma parte da responsabilidade de aprender. Fico pensando em, como fez a Tâmara Milhomem, dar essa tecnologia para os alunos construírem conteúdos. É com certeza, uma experiência muito rica e multidisciplinar.

\section{Referências}


CAZDEN, Courtney; COPE, Bill; FAIRCLOUGH, Norman; GEE, Jim; et al. A pedagogy of multiliteracies: Designing social futures. Harvard Educational Review, v. 66, n.1, 1996.

COSCARELLI, Carla Viana. Receitas sobre pesquisa em leitura e letramento digital: entrevista com Carla Viana Coscarelli. Educação \& Tecnologia, Belo Horizonte, v. 14, n. 1, p. 4-9, jan./abr. 2009. Disponível em: <https://seer.dppg.cefetmg.br/index.php/revista-et/article/view/206/206\#>. Acesso em: 17 out. 2020.

COSCARELLI, Carla Viana; AMARAL, Luana Lopes. O ensino de escrita acadêmica a distância na UFMG: um relato de experiências. Revista Docência do Ensino Superior, [S. l.], v. 7, n. 2, p. 182-199, 2017. Disponível em: <https://periodicos.ufmg.br/index.php/rdes/article/view/2340>. Acesso em: 19 out. 2020 .

COSCARELLI, Carla Viana; RIBEIRO, Ana Elisa. Leitura e ensino: por avaliações que levem (mesmo) os ambientes digitais em consideração. Texto Digital, Florianópolis, v. 15, n. 2, p. 101-129, 2019. Disponível em: $<$ https://periodicos.ufsc.br/index.php/textodigital/article/view/18079288.2019v15n2p101/42347>. Acesso em: 16 out. 2020.

COSCARELLI, Carla Viana; RIBEIRO, Ana Elisa. O que dizem as matrizes de habilidades sobre a leitura em ambientes digitais. Educação em revista, Belo Horizonte, v. 26, n. 3, p. 317-334, dez. 2010. Disponível em:

<https://www.scielo.br/pdf/edur/v26n3/v26n3a16.pdf>. Acesso em: 18 out. 2020.

KIRNER, Claudio; SISCOUTTO, Robson A. Fundamentos de Realidade Virtual e Aumentada. In: KIRNER, Claudio; SISCOUTTO, Robson A. (Eds). Realidade Virtual e Aumentada: Conceitos, Projeto e Aplicações. Petrópolis, RJ, Editora SBC - Sociedade Brasileira de Computação, Porto Alegre, 2007.

KRENAK, Ailton. Ideias para adiar o fim do mundo. São Paulo: Companhia das Letras, 2019.

KRESS, Gunther, VAN LEEUWEN, Theo. Multimodal Discourse: the modes and media of contemporary communication. London: Edward Arnold, 2002.

KRESS, Gunther, VAN LEEUWEN, Theo. Reading Images. The Grammar of Visual Design. London: Routledge, 1996.

RANCIÈRE, Jacques. O mestre ignorante: Cinco lições sobre a emancipação intelectual. Trad. Lílian do Valle. Belo Horizonte: Autêntica, 2002.

RIBEIRO, Ana Elisa. Textos Multimodais: leituras e produções. São Paulo, Parábola Editorial, 2016. 
Thayane Verçosa, Marcela S. Brigida e Gabriela R. Nunes, Entrevista com Carla Coscarelli

SOARES, Magda. Letramento: um tema em três gêneros. Belo Horizonte: Autêntica, 1998. 


\title{
Digital Literacy and Multimodality: An Interview with Professor Carla Coscarelli in Pandemic Times
}

\author{
Carla Viana Coscarelli ${ }^{\mathrm{v}}$
}

Interviewers:

Thayane Verçosa ${ }^{\text {vi }}$

Marcela Santos Brigida ${ }^{\text {vii }}$

Gabriela Ribeiro Nunes ${ }^{\text {viii }}$

Translated By

Marcela Santos Brigida

The impressive professional history of Professor Carla Viana Coscarelli, one that is centered on themes such as reading, text production, teaching and digital literacy, justifies, on itself, her presence as an interviewee in the present issue of Palimpsesto. She holds a BA in Literature (Federal University of Minas Gerais UFMG), as well as an MA and a PhD in Linguistic Studies (UFMG). Coscarelli was a postdoctoral fellow in Cognitive Sciences at the University of California (San Diego) and in Education at the University of Rhode Island. Her doctoral thesis, "Leitura em ambiente multimídia e a produção de inferências" ["Reading in Multimedia Environments and the Production of Inferences"] (1999), is an example of the studies developed by the interviewee on the inclusion of multiple sources in the process of reading and learning. Those are investigations she has been developing continually throughout her career. Coscarelli is currently a Professor at the Federal University of Minas Gerais (UFMG), where she is a member of

\footnotetext{
${ }^{v} \mathrm{PhD}$, Linguistic Studies at Federal University of Minas Gerais (1999); Postdoctoral fellow in Cognitive Sciences at the University of California San Diego (2005); Postdoctoral fellow in Education at the University of Rhode Island. Professor at Federal University of Minas Gerais (UFMG).

${ }^{\text {vi }} \mathrm{PhD}$ Student in Literary Studies at UERJ. CAPES Scholar. thayanevercosa@ hotmail.com

vii PhD Student in English Literature at UERJ. CNPq Scholar. marcela.brigida@uerj.br

viii PhD Student in Literary Theory at UERJ. j15c17g11@ @otmail.com
} 
the Lingtec Research Center (Language and Technology), of Ceale (Center for Literacy, Reading and Writing). She also coordinates the Redigir Extensionist Project at UFMG, a wonderful example of how teaching, research and extensionist activities, when wellarticulated, can generate extremely fruitful results.

It is with great satisfaction that we bring you this interview with Professor Carla Coscarelli, who promptly and kindly accepted our invitation, being very receptive to the questions we proposed. We would like to highlight that it was a pleasure to get to know more of the professor's inspiring career history and to learn from her production on topics that are extremely relevant, especially in the current context of global diffusion of emergency remote teaching.

\section{PALIMPSESTO}

1) Eleven years ago, in your interview with the journal Educação \& Tecnologia, the question "What happens to literacy in times of web access and digital social networks?" was answered as follows: "I think literacy can do well in digital times. The web today is very dependent on writing and reading. This may change, but today, we need to be literate to resourcefully deal with the computer and the web. This creates a demand and people tend to react to these social demands, learning what they need to know, whether at school, or with friends and relatives. The need to read and write may create a desire to learn and having real situations that require reading and writing texts will help in developing the skills necessary for both processes. I believe that schools are preparing to help people to handle those environments well and I hope that this will soon not be a privilege of the upper classes, but also a reality of the popular strata" (COSCARELLI, 2009 , p. 7). In view of what has changed over more than a decade and considering that since then the presence of the web and of social networks in daily life has intensified, how would you answer that same question today?

\section{CARLA COSCARELLI}

On the one hand, it is good to say that I would respond it quite similarly. Literacy, which is a part of this endless process of conquering the state or condition developed by those who appropriate writing (SOARES, 1998), is particularly important in our society. Computers, including mobile phones, which are actually pocket computers, are part of our lives, and writing is required in most of the actions we take on these digital devices. 
I would add there the work with multimodality, that is, work with the various languages, in addition to the increasing need to pose a critical and attentive eye to all the information we find, so that we can detect and deal with fake news, to avoid falling victims to scams, which are growing increasingly sophisticated.

Literacy must be seen in a comprehensive way, because it needs to incorporate writing and reading into those equipment, for example, to know how to use the keyboard, to know how to get familiar with text-production programs such as text editors (e.g. Word), slide-presentation programs (e.g. PowerPoint), programs for producing infographics, banners, social media posts, spreadsheets.

The production of such texts requires knowledge of how to use equipment (e.g. computers, mobile phones, keyboards, a mouse, programs, applications) that must be thoroughly known and, as far as possible, mastered by users. The computer is our notebook, and we must learn to write on it, exploring the resources it offers us.

Digital information and communication technologies have brought new spaces for sharing texts, new spaces for reading and writing, new forms of communication in which audios, videos, animations, images, photographs are shared. So to know how to receive (to read, to see, to watch, to appreciate, to critique, etc.), to know how to produce and to share these texts are skills that we must have as citizens in our contemporary society. To deny this to people is to deny access to information and, in some way, it is also to deny the right to freedom of speech, which is against the law.

On the other hand, it is sad to say that my answer to this question remains practically the same. After more than a decade, I would like to say that schools have prepared themselves and that today people know how to use digital environments well, that they are good readers and producers of the textual genres that circulate there and that they know how to deal well with the different languages that they demand or provide. I would also say that access to digital and internet equipment is equal for all and that schools are places where you learn to deal with them in a respectful, ethical, creative, and emancipatory way. Unfortunately, I will have to continue wishing that schools will prepare people to deal with these environments well and argue that we need to fight hard so that access to equipment and information is no longer a privilege of the upper classes of our society and becomes a right (that is respected and fulfilled) of all. 


\section{PALIMPSESTO}

2) In the context of a pandemic and social isolation, in which our forms of social interaction, work and teaching were directly and, perhaps, irreversibly impacted, what is your opinion on the current massive implementation of distance learning, considering that it, in most cases, has consisted in an attempt to transfer the classroom structure to various digital platforms? Is it possible to think of the advantages and disadvantages of this process of transference?

\section{CARLA COSCARELLI}

First, we need to establish a distinction between distance learning and emergency remote education. Distance learning has regulations. Those are (or should be) wellplanned courses, in which a virtual learning environment (VLE) is chosen so that classes will take place in it. Professors are prepared for this type of teaching, they know how to deal with VLE, they plan didactic strategies to approach the subjects in this teaching modality. They plan the forms of interaction with the students in each activity, and they balance synchronous and asynchronous activities depending on the demands of the course and classes. Those professors know beforehand that they need to have certain equipment such as a good computer, microphones, cameras; they need to have a good and stable internet connection. In addition, they know how to deal with programs to record and edit classes or they have a structure to assist the recording and editing of audio and/or video files. They know how to create activities, evaluations, forms of interaction between participants, that is, they know how to plan and manage virtual learning spaces. The students, in turn, know that the classes will take place remotely, and therefore they need to have the equipment to access the activities, to carry out the tasks and to participate in the interactions. Students also need to be organized and disciplined learners and, when enrolling, they know and accept that the course will take place in a distance learning modality. It is a choice. There is a pre-established preparation and agreement between those who participate in distance learning.

Emergency remote education, in turn, was the result of a situation that prevented face-to-face meetings caused by the pandemic. Suddenly, face-to-face courses could not be taught in this format and other forms of contact with students had to be created. Some schools and communities were able to use computers almost immediately to continue their work. Others were unable to do this because students and professors did not have 
the equipment and resources to establish this transfer from one modality to the other. This is a delicate issue, one that reflects a society of great socioeconomic differences between people and that demands a great deal of discussion about structural issues in our society and about public policies. So, I will stick to your question: trying to transfer exactly what happens in the classroom to digital platforms is a big mistake, because the dynamics must be different. In the classroom, the teacher, for better or for worse, can give a 40-minute lecture. Online, students will turn off their cameras and do other things. In a face-to-face class, students exchange ideas with the classmate next to them, talk between one class and the other, have relaxation and interactions with colleagues at break time. So, we need to think of ways to keep interactions between students going.

Classes in digital environments must be designed for this format, which makes it possible, for example, for activities to be asynchronous (synchronous - everyone in the same "place" at the same time / asynchronous - each person works at the time that suits them best). In a face-to-face setting, what has taken place does not rewind. Online, I can watch the video several times, I can perform the activity at a different time than that stipulated by the school. This possibility needs to be considered, because we do not know if at a given home there are two students with classes in the morning and only one computer available, for example. In that case, someone would be harmed. We do not need to call the roll because VLE tells me who entered the environment and what activities this student performed there. In that same environment, the professor can read the work done by the students, send feedback, mark papers; other students can read and comment on the work of their colleagues and, if applicable, students can review what they produced by incorporating the comments received, reposting they production. Those are dynamics that can be explored in asynchronous teaching, and that do not depend on everyone being together in a virtual classroom at the same time. Synchronous meetings can also be interesting, productive, and enriching, but it depends on what will be developed in them. Long lectures and teacher-centered dynamics may not be the most productive way to use the time for synchronous activities. The time with students (which need not be the total course load) should be used to talk about the tasks, for students to bring and discuss doubts, to share results. From a first conversation and a proposal made and negotiated with the class in a synchronous meeting, groups of students can, during asynchronous activities, carry out the task by talking, writing and producing the work collaboratively 
using digital environments (e.g. WhatsApp, Google Docs, tools available in the adopted VLE).

I adopt in my thinking and in my practices a conception of learning in which students carry out activities to learn under the guidance of the professor. I like studentcentered education and know that it is very productive.

\section{PALIMPSESTO}

3) In the article you published with Professor Ana Elisa Ribeiro, "Leitura e ensino: por avaliações que levem (mesmo) os ambientes digitais em consideração" (2019), when reflecting on the growth and relevance of reading in digital environments, as well as justifying the need for reading practices in such environments to be included in school curricula, you observe: "Most teenagers seem to never have learned how to go about judging the reliability of the information they find on the internet [...], since they do not use efficient strategies to assess this issue" (COSCARELLI; RIBEIRO, 2019, p. 103). To what extent is this educational gap related to the spread of fake news and its negative impact on people's daily lives? In addition to including these practices in school curricula, can we think of other measures to help build the capacity to "judge the reliability of information"?

\section{CARLA COSCARELLI}

The issue of fake news is complex, because there is the spread of false news, but there are those who benefit and those who support the dissemination of such news because they reflect and reinforce an ideology, the ideas of a certain group, a set of behaviors that some people want to reinforce, etc. But, in any case, being a critical and attentive reader is directly related to the spread of fake news, since this type of reader can help slow the spread of misleading news and influence others to examine more carefully the information they receive.

People need to analyze the information considering who said it, in what situation, if that person has competence and expertise to deal with that topic, who is interested in that information, what voices or aspects are silenced in it, that is, they have to think about that material with caution and a certain degree of meticulousness. You have to be suspicious and look for what is behind that speech, what is between the lines, why that was said that way, if those arguments are robust, if evidence is presented, if that 
information is true, what would be the sources to that information, among many other questions that can be asked.

People need to learn to read critically, to reflect on the information they find or receive, and to assess the relevance of that information before passing it on to others. This involves a reading that we can call a deeper reading as opposed to a superficial reading without reflection. No reading should be superficial, should it? There is no unassuming or neutral text, no discourse that is not rhetorical and argumentative. So, the reader must get used to doing this exercise of suspicious reading.

The ability to assess the reliability of information must always be encouraged by schools, by parents, and in campaigns by the most diverse media outlets. It is a matter of social responsibility and safety, as this suspicious reading can also serve as prevention against scams and crimes that are increasingly frequent in digital environments.

\section{PALIMPSESTO}

4) In the text you published with Luana Lopes Amaral, "O ensino de escrita acadêmica a distância na UFMG: um relato de experiências" (2017) ["The remote teaching of academic writing at UFMG: a report of experiences"] (2017), the positive results of the course "Oficina de Língua Portuguesa: Leitura e Produção de Textos" ["Portuguese Language Workshop: Reading and Text Production"] are evident. It was taught in distance learning (via Moodle) and offered to all undergraduate students at UFMG for over a decade. When thanking the partnerships signed with different organs at the University, you said: "There is still a lot of prejudice and mistaken ideas regarding distance learning, and this support from higher levels at the university has helped us to show that this teaching modality is as efficient and suitable as face-to-face teaching several research data point to this fact [...]. In addition, it has enabled us to explore the diverse potentialities of this teaching modality, developing cutting-edge work at the university, which can serve as a model and experience for other teachers and courses" (COSCARELLI; AMARAL, 2017, p. 194). In your opinion, what is the reason for this prejudice in relation to distance learning? To what extent did the "Workshop" contribute to overcoming it at the university where you work? Are you aware of similar initiatives, influenced or not by the "Workshop"?

\section{CARLA COSCARELLI}


I cannot say that every distance learning course is great, just as I cannot argue that every face-to-face course is of good quality. We know that there are good and bad courses in both modalities.

Prejudice in relation to distance learning currently comes from poor information about how these courses take place. However, if we think about the history of distance learning, this prejudice may have come from the slow exchange of information by mail in the past, in courses that were conducted by correspondence. Classes took a long time to reach the student, their response took a long time to reach the teacher, feedback and evaluations of the work arrived when the student probably could not even remember anymore what he sent because of the slow form of communication. In addition, it was a solitary way of learning, without a class or a group of people who exchanged ideas quickly and easily. The student needed to have a lot of motivation and patience to learn. Therefore, many people gave up, which ended up being regarded as a fault in the course. We may also consider economic factors, that is, who had money would go to university, who had fewer resources had to be content with distance learning courses via correspondence or the radio. So, due to a series of factors, distance learning was stigmatized as a modality of smaller, worse, ineffective teaching, but, in fact, we know that it served millions of students who learned their professions or a trade (Instituto Universal Brasileiro, Rádio- Municipal School in Rio, among others).

With the internet, the scenario for distance learning changes. Communication becomes faster and is increasingly accessible to a greater number of people, which makes the establishment of groups of interest easy, as well as synchronous meetings or the exchange of messages and materials asynchronously. This makes the courses more dynamic. Now they can explore different types of materials such as texts, audios, videos, animations, discussion forums, among many other resources that can easily be used.

The Reading and Text Production Workshop works with the insertion of students in the academic universe and, with that intent, it presents Currículo Lattes and Portal de Periódicos da Capes to students, discussing the different ways of citing works and the gravity of plagiarism in the academy. It involves the production of abstracts, reviews, the writing of a project, the collection of data, the analysis of the data and the preparation of a small research report. This is an important initial training for students and ends up having a positive impact on all their educations at the university, as the students 
themselves report. The material and dynamics of the Workshop have been built and improved every semester for more than ten years. Therefore, we ended up finding a very robust (but not formulaic) way of working, which has been evaluated very positively by the students. We serve 500 students from different UFMG courses per term and have a very dynamic and well-integrated team with teachers and tutors.

At the Faculty of Letters at UFMG we have other extraordinarily successful projects such as teaching English (Ingrede), which started at the same time as our Workshop, and teaching Brazilian Sign Language, which is a little more recent. There are many other beautiful distance learning projects around Brazil. The Universidade Aberta do Brasil [Open University of Brazil], for instance, is a program that has greatly expanded the offer of distance learning for higher education. We must hope there are no plans to shut it down.

\section{PALIMPSESTO}

5) In the text "O que dizem as matrizes de habilidades sobre a leitura em ambientes digitais" (2010) ["What the skills matrices say about reading in digital environments"], you and Professor Ana Elisa Ribeiro discussed "aspects of the Portuguese language matrix used as a basis for the composition of basic education tests", reflecting, to a large extent, on "how this matrix is [i] impregnated with values linked to the written print culture; [ ii ] based on assumptions that do not take into account the multimodality of texts that circulate in society; [iii] imprecisely written in a large portion of the descriptors; and [iv ] lacking an extension to add important elements for a less vague assessment of skills supported by reading experiences in a digital environment" (COSCARELLI; RIBEIRO, 2010, p. 318). A decade later, did the skill matrices on reading come to include elements of assessment that effectively contemplate reading in a digital environment? What were the advances? What about the challenges yet to be faced?

\section{CARLA COSCARELLI}

We can say that the National Curricular Base (BNCC) was a breakthrough, regarding the inclusion of the digital element in pedagogical proposals. The Base expresses a concern with diversity and with the training of subjects to exercise citizenship. The Base explicitly speaks of Digital Information and Communication Technologies (DICT) and mentions many digital genres such as meme, vlog, podcast, digital cartoon, among others. 
In addition, BNCC talks about navigation, although without mentioning that word. It talks about searching and selecting information and even mentions curatorship, which we can understand as the construction and organization of a collection or a repertoire with information that will be useful or necessary for a given purpose. It talks about selecting information from trusted sources, both in print and digital form. This document presents the important skills for dealing with digital technologies as a transversal aspect, which crosses several areas and themes. That is good.

The Base mentions many textual genres that will demand the use of equipment such as recorders, camcorders, cameras, microphones, audio, video and written text editors, and all of this is on a mobile phone, which is prohibited in many schools. So, I think that BNCC forces us to rethink the school and its ways of doing education, pushing for a more contemporary education, which will teach students to use the equipment they bring in their pockets to learn, to produce content and share their creations.

BNCC also has a concern with multimodality, with the exploration of languages other than verbal. The document explicitly mentions practices that involve the reception, production and interaction made with multisemiotic and multimedia texts. This is an improvement over other evaluation matrices, such as the Basic Education Evaluation System (SAEB).

We know, however, that theory is not always put to practice. For the ideas presented in the Base to be executed, it is necessary that education professionals receive training for this and can put these ideas into practice. It is essential that the school community can count on good equipment, good internet access (including at home, where part of the work is done by both teachers and students). This pandemic showed us that we are far from having the basics to think about education for digital times, especially in public education, which represents more than $70 \%$ of the school population in Brazil.

\section{PALIMPSESTO}

6) The Redigir Project, which is coordinated by you, has since 2009 made available, to be used in Portuguese language classes of basic education, activities aligned with contemporary theories of linguistic, literary, discursive and education studies. How does the articulation between theory and practice take place in the development of these activities? Does the project consider the changes in the official documents of the Brazilian 
Ministry of Education aimed at defining parameters for the teaching of the Portuguese language in the country? In more than ten years of activity, how have the contact and the dialogue between the University and the school community using the materials produced by the project been?

\section{CARLA COSCARELLI}

Redigir completed 21 years of activities in 2020. In this project, we look forward to put into practice a conception of Portuguese language teaching that is more focused on literacy, that is, on the use of languages and not on metalanguage pure and dry, as it happens in the normative teaching of traditional grammar, which is still quite common in our schools.

We create activities to be applied by teachers, thinking about themes, textual and discursive genres that are important for the formation of a contemporary citizen. Therefore, we work with themes such as the diaspora, cities, ethnicities, racism, violence against women, consumption, relationships, art, graffiti. There are many subjects. We work with different textual genres such as songs, short films, poems, short stories, chronicles, product packaging, points of sale, advertisements, news pieces, among many others. It is a project that seeks to treat the topics in a light and interesting way for students and teachers, without failing to discuss the topics with seriousness and depth.

We seek to develop skills related to digital literacy and, for this, we bring activities that deal with the search, selection, analysis, synthesis, integration and use of information from multiple sources, with the use of digital technologies available for free on the internet.

We study theories from various areas, and which are important for thinking about life. Those are theories that help us to understand education, languages, people, societies, media, the digital universe, and its influence on our lives. Being attentive to these theories, we can think of ways to work the texts with the students, bringing the interdisciplinary exploration of the themes and pointing out aspects that need to be perceived, analyzed and evaluated by the students. Theories help guide our view. For example, we always have inspiring theorists from different areas, such as Magda Soares, Paulo Freire, Celestin Freinet, Mimi Ito, Sugata Mitra, Jean François Rouet, Jacques Ranciere, Ailton Krenak, Djamila Ribeiro, Gunther Kress, Edgar Morin, Roxane Rojo, 
Ana Elisa Ribeiro, Mário Perini, John Dewey, Vygotsky, Wlakiria Monte Mór, Julie Coiro, Renée Hobbs, Roxane Rojo, among others. It is a large group of researchers and thinkers that inspire us, and it is a list that grows every day because we are always reading, researching, and seeking contributions from studies in different areas.

The Multiliteracies theory, proposed by the New London Group (Cazden et al., 1996), reminds us that we need to think about the multiple languages orchestrated in a text and that texts are always multimodal (KRESS, VAN LEEUWEN, 1996, 2002; RIBEIRO, 2016). Even when they seem to be made up only of words, there is a choice of font, colors, layout, spacing, formatting in general, which, in themselves, already bring meanings to be constructed by readers. The programs and applications available in digital environments bring many possibilities for working with various languages, making it increasingly simple to explore visual resources in texts, to produce and combine audios, videos, animations.

Multiliteracies also call our attention to the cultural diversity of our societies, which must be understood and respected. I will give the example of the linguistic variety. We are used to thinking that in Brazil we all speak the same language. This is not true, we have several indigenous, quilombola languages. In some communities, people speak oriental languages, in others they speak European languages or dialects of them. And even within Portuguese, we have a vast range of registers and variants that must be known, respected and destigmatized. Linguistic prejudice exists. It generates discrimination, and, like all types of prejudice (religious, racial, social, among others), it needs to be tackled, because it is a way of putting some people in an inferior position so that others assume a role superiority, triggering a very cruel power process.

In many situations, there is an attempt to erase people's accents (see for example the television media / record companies). This is a very silent and symbolic violence. It is the silencing of voices, cultures, and differences for the installation of a "higher standard" that makes it so that everything that is different from that is perceived as inferior, bad, worthy of being ignored and exterminated. It is hideous, inhuman, and extremely dangerous.

The notion of Multiliteracies is very dear to us because it gives us a view that we are diverse, multiple, that different cultures manifest themselves in different combinations 
of languages and it is very impoverishing to choose only one form or culture as the one that needs to prevail over others.

We prefer emancipation to brutalization (RANCIERE, 2002, KRENAK, 2019). Therefore, in our activities we seek ways to give autonomy to students, working on questioning, on the critical eye. We try to give them a voice and develop ways of empowerment, of understanding social phenomena, of fighting prejudices, etc.

There are many theories at stake, I do not have space here to talk about all of them, but I hope I have shown the spirit of the theories that guide our work.

We think a lot about the teachers as well, the activities are a way to develop their continuing education. All our activities have quite a detailed section for the teacher, establishing a dialogue between our team and them, showing possibilities for answers, guidance, presenting possibilities and explaining what we intend with those questions. We strive for activities to help teachers move out of the traditional format and to think that mother-language teaching can be done in alternative ways, that linguistic reflection can (and should) be done outside the bonds and inconsistencies of traditional grammar. It can be done in a contextualized and meaningful way for students.

We increasingly receive responses and testimonials from teachers who use our activities. We also received suggestions for themes for the activities. We try to understand and meet their demands. At the beginning of the pandemic, for example, realizing that many teachers were tense, full of doubts and questions, we created a series of tips that showed applications and programs that they could use with students and gave them suggestions on how to do this. Those tips were gathered in an e-book that is available to teachers for free (like everything we make for them) on our website (www.redigirufmg.org).

We have an intense dialogue with teachers through social media. With the pandemic and physical isolation, we received many invitations to talk to teachers from schools in different regions of the country. We had many livestreams that were extraordinarily rich conversations with teachers. Education faces an even more challenging situation in this pandemic. That would add many more pages of conversation, which I will summarize by saying that the university and the community are much closer partners than people realize. The public university has numerous partnerships with the community both directly in its projects and extensionist programs, as well as in the 
research that generates a positive impact on people's lives. Not to mention teaching, which completes our tripod (teaching, research, and extensionist activities), and that returns trained professionals to work in the most diverse areas to society. Yes, I am passionate and enthusiastic about the public university. I am the result of public education; I believe and defend public education of high quality for all.

\section{PALIMPSESTO}

7) Your most recent research project "Realidade aumentada: buscando formas de promover a leitura e a aprendizagem" ["Augmented reality: looking for ways to promote reading and learning"], which took off in 2019, is described by you as follows: "The main goal of this project is to develop ways to use augmented reality to promote autonomous learning and critical reading. For this, we will produce text comprehension materials that will serve as a guide for readers. These materials aim to work on reading skills that are essential for understanding multimodal texts from different genres and discursive domains" (Source: Currículo Lattes). What is augmented reality and how can it assist in promoting autonomous learning and critical reading? How will the materials serve as a guide for readers? How does augmented reading work in practice?

\section{CARLA COSCARELLI}

We have a lot of digital technology available and we need to think about its potential. In my case, I look for ways to improve people's reading skills, to help students become fluent, critical, and competent readers. Augmented reality is, roughly speaking, a way of integrating the virtual and the real. It is a way to enrich or complement "the real environment with virtual objects, using some technological device, working in real time". This is a definition by Kirner and Siscoutto (2007, p. 10).

It is a technology that activates links from images, it is an extension of the hypertext. The QR code, for example, is a simple form of augmented reality. That image takes you to other places. Pokémon Go, which was a massive success, uses augmented reality. From a trigger - an image recognized by a camera or sensor, which in turn may be on a phone, on a tablet, on glasses - you can activate audio, animation, film files, and other sources of information. This creates enormous possibilities. You can, by recognizing the facade of a house, see a character telling some historical fact that happened there. From a poster, you can see the artist talking about the show and inviting you to watch it. With the image of a cell as a trigger, you can appreciate the ways in which 
it reproduces. When you identify a work of art with your mobile phone, you can see the artist producing that work, talking about it, or get some information about the artist, the time and circumstances in which it was produced, as well as some tips on how to interpret or better understand that piece. From a work of graffiti, you can zoom in and animate that image and "enter" it, experiencing, in a very real and corporeal form, that new reality, as in the art project Aquário Urbano by Felipe Yung Maciel (Flip) and Kléber Pagu, who developed a virtual aquarium based on a work of graffiti painted on buildings in São Paulo. A wonderful project.

The potential of this technology is enormous in the most diverse areas of knowledge and it can be a strong ally in education. Students can learn a lot with augmented reality and can produce materials using this resource, which will generate very enriching interdisciplinary knowledge.

Tamara Milhomem has developed quite an interesting project in the UFPI library, with her students from Instituto Federal do Piauí - Campus Picos, joining the desire to encourage reading, to develop text interpretation skills and augmented reality. She encouraged students to create augmented reality from the covers of books they read. The students selected the works, read, discussed and created the augmented reality - from the cover, the back cover or the spine of the book - that triggered a production of those students based on the book, as a way to motivate other students to read that work. It was a success.

I am developing with the Interaction team at USACH, under the coordination of Professor Roberto Gonzáles Ibañez, a project that uses augmented reality to develop reading skills. It consists of integrating print and digital formats. From the recognition of the text and movements made with hands, the readers can complement the text with extra information, such as the meaning of words, encyclopedia entries, images, animations, films, or explanatory audios. They can make notes that will be stored on the computer and/or view notes made by others. You can select and save parts of the text, among other possibilities.

We are also developing another project in which the reader uses a mobile phone to, from the printed text, activate images, animations and audios that bring questions or information about elements found on that page, exploring the images of the text as well as its verbal part. Thus, the augmented reality application will show the student the 
questions he can ask the texts, the information he may want to look for outside of it. He can perceive the importance of articulating verbal and visual resources, the inferences he can or needs to do to enrich the reading and the questioning that will lead to a critical reading of the materials that he will find in the most diverse environments and communicative situations. Thus, in a light way, we try to develop the subjects' reading competence. Augmented reading, as we like to call it, can be used by people of all ages, if appropriate materials are made for that age group and for those specific purposes and themes.

This can all be used for educational purposes and to promote learning. The user, with such material, can learn to learn. I would like to remind you that the presence of the teacher is always important, as he will promote discussions, he will guide students in their learning, he will organize the workload, select topics and think about gradations and difficulties, and launch challenges. Such a program does not exempt the teacher, quite on the contrary; the teacher is an important piece to guide students in the use and creation of these materials.

I think it is very important for students to do things, to produce materials, to be active in learning, so the teacher must deliver to the students the possibility of building their own ways of learning and part of the responsibility to learn. I keep thinking, as did Tâmara Milhomem, in giving this technology for students to build content. It is certainly an extraordinarily rich and multidisciplinary experience.

\section{Works Cited}

CAZDEN, Courtney; COPE, Bill; FAIRCLOUGH, Norman; GEE, Jim; et al. A pedagogy of multiliteracies: Designing social futures. Harvard Educational Review, v. 66, n.1, 1996.

COSCARELLI, Carla Viana. Receitas sobre pesquisa em leitura e letramento digital: entrevista com Carla Viana Coscarelli. Educação \& Tecnologia, Belo Horizonte, v. 14, n. 1, p. 4-9, jan. /abr. 2009. Available at: https://seer.dppg.cefetmg.br/index.php/revistaet/article/view/206/206\#. Accessed on: 17 Oct. 2020.

COSCARELLI, Carla Viana; AMARAL, Luana Lopes. O ensino de escrita acadêmica a distância na UFMG: um relato de experiências. Revista Docência do Ensino

Superior, [S. l.], v. 7, n. 2, p. 182-199, 2017. Available at:

https://periodicos.ufmg.br/index.php/rdes/article/view/2340. Accessed on: 19 Oct. 2020. 
COSCARELLI, Carla Viana; RIBEIRO, Ana Elisa. Leitura e ensino: por avaliações que levem (mesmo) os ambientes digitais em consideração. Texto Digital, Florianópolis, v. 15, n. 2, p. 101-129, 2019. Available at:

https://periodicos.ufsc.br/index.php/textodigital/article/view/1807-

9288.2019v15n2p101/42347. Accessed on: 16 Oct. 2020.

COSCARELLI, Carla Viana; RIBEIRO, Ana Elisa. O que dizem as matrizes de habilidades sobre a leitura em ambientes digitais. Educação em revista, Belo Horizonte, v. 26, n. 3, p. 317-334, dez. 2010. Available at:

https://www.scielo.br/pdf/edur/v26n3/v26n3a16.pdf. Accessed on: 18 Oct. 2020.

KIRNER, Claudio; SISCOUTTO, Robson A. Fundamentos de Realidade Virtual e Aumentada. In: KIRNER, Claudio; SISCOUTTO, Robson A. (Eds). Realidade Virtual e Aumentada: Conceitos, Projeto e Aplicações. Petrópolis, RJ, Editora SBC - Sociedade Brasileira de Computação, Porto Alegre, 2007.

KRENAK, Ailton. Ideias para adiar o fim do mundo. São Paulo: Companhia das Letras, 2019.

KRESS, Gunther, VAN LEEUWEN, Theo. Multimodal Discourse: the modes and media of contemporary communication. London: Edward Arnold, 2002.

KRESS, Gunther, VAN LEEUWEN, Theo. Reading Images. The Grammar of Visual Design. London: Routledge, 1996.

RANCIÈRE, Jacques. O mestre ignorante: Cinco lições sobre a emancipação intelectual. Trad. Lílian do Valle. Belo Horizonte: Autêntica, 2002.

RIBEIRO, Ana Elisa. Textos Multimodais: leituras e produções. São Paulo, Parábola Editorial, 2016.

SOARES, Magda. Letramento: um tema em três gêneros. Belo Horizonte: Autêntica, 1998. 